\title{
REGULAR IDENTITIES IN LATTICES
}

\author{
BY \\ R. PADMANABHAN $\left({ }^{1}\right)$
}

\begin{abstract}
An algebraic system $\mathfrak{A}=\langle A ;+, 0\rangle$ is called a quasilattice if the two binary operations + and $\circ$ are semilattice operations such that the natural partial order relation determined by + enjoys the substitution property with respect to $\circ$ and vice versa. An identity " $f=g$ " in an algebra is called regular if the set of variables occurring in the polynomial $f$ is the same as that in $g$. It is called $n$-ary if the number of variables involved in it is at the most $n$. In this paper we show that the class of all quasilattices is definable by means of ternary regular lattice identities and that these identities span the set of all regular lattice identities and that the arity of these defining equations is the best possible. From these results it is deduced that the class of all quasilattices is the smallest equational class containing both the class of all lattices and the class of all semilattices in the lattice of all equational classes of algebras of type $\langle 2,2\rangle$ and that the lattice of all equational classes of quasilattices is distributive.
\end{abstract}

An algebra $\mathfrak{U}=\langle A,+, \circ\rangle$ with two binary operations + and $\circ$ is called a quasilattice if both + and $\circ$ are semilattice operations in $A$ such that the natural partial order relation determined by + enjoys the substitution property with respect to $\circ$ and vice versa. Thus every lattice is a quasilattice. In this paper we study the axiomatic significance of the class of all quasilattices. An equation " $f=g$ " in an algebra is called regular if the set of variables occurring in the polynomial $f$ is the same as that in $g$. It turns out that the class of all quasilattices is equationally definable and that the defining equations are all regular (Lemma 1) and that these equations span the set of all regular equations true in all lattices (Theorem 1). The proof is via a representation theorem for quasilattices as the sum of a direct system of lattices (Lemma 3). From this result it is deduced that the class of all quasilattices is the smallest equational class containing both lattices and semilattices (in a prescribed sense) in the lattice of all equational classes of algebras of type $\langle 2,2\rangle$ (Theorem 2) and that the set of all binary regular lattice identities (i.e. regular identities involving at the most two elements) is finitely based. The essential tool we make use of is the concept of a partition function in algebras, which is due to J. Plonka.

Presented to the Society, February 17, 1969 under the title Regular equations in lattices and July 28, 1969; received by the editors July 26, 1969.

AMS 1969 subject classifications. Primary 0242, 0254; Secondary 0630.

Key words and phrases. Bi-semilattices, quasilattices, Birkhoff systems, regular identities, compatibility property, partition function, equational class, types of algebras, strictly consistent, base.

(1) This research was supported by the National Research Council of Canada.

Copyright (C) 1971, American Mathematical Society 
1. Notations and definitions. Let $\langle A, *\rangle$ be a semilattice (i.e. $*$ is an idempotent, commutative and associative binary operation in $A$ ). Then by $R_{*}$ we mean the natural partial order relation determined by $*$, namely,

$$
R_{*}=\{(a, b) \mid a \in A, b \in A, a * b=a\} .
$$

A binary relation $R$ in $A$ is said to be compatible with a binary operation $*$ defined in $A$ if

$$
(a, b) \in R \Rightarrow(a * c, b * c) \in R \quad \text { and } \quad(c * a, c * b) \in R \text { for all } c \in A .
$$

It is to be noted that if $R$ is reflexive and transitive then the above definition of compatibility is equivalent to the following substitution property:

$$
(a, b) \in R,(c, d) \in R \Rightarrow(a * c, b * d) \in R .
$$

An algebra $\mathfrak{A}=\langle A,+, \circ\rangle$ is called a quasilattice if + and $\circ$ are two semilattice operations in $A$ such that the natural partial order relation determined by + is compatible with $\circ$ and vice versa. In other words, an algebra $\mathfrak{A}=\langle A,+, \circ\rangle$ will be a quasilattice if and only if the following conditions hold in it:

Q1. $a+a=a$,

Q2. $a+b=b+a$,

Q3. $a+(b+c)=(a+b)+c$,

Q4. $a+b=a \Rightarrow a c+b c=a c$

and their duals $\mathbf{Q 1}^{\prime}, \ldots, \mathrm{Q} 4^{\prime}$.

If $\mathfrak{A}=\langle A,+, \circ\rangle$ satisfies $\mathrm{Q} 1, \mathrm{Q} 2, \mathrm{Q} 3$ and their duals and

$$
a+b a=(a+b) a
$$

then it is called a Birkhoff system( $\left.{ }^{2}\right)$.

Let $\mathfrak{A}=\langle A, F\rangle$ be a universal algebra. J. Plonka has introduced the notion of a partition function in $\mathfrak{A}$ which is a binary function $f: A^{2} \rightarrow A$ satisfying the following conditions (see Lemma 1 of [8]):

P1. $f(x, x)=x$,

P2. $f(x, f(y, z))=f(x, f(z, y))$,

P3. $f(x, f(y, z))=f(f(x, y), z)$,

P4. $f\left(f_{n}\left(x_{1}, \ldots, x_{n}\right), y\right)=f_{n}\left(x_{1}, \ldots, x_{i-1}, f\left(x_{i}, y\right), x_{i+1}, \ldots, x_{n}\right), i=1, \ldots, n$,

P5. $f\left(x_{1}, f\left(x_{2}, \ldots, f\left(x_{n}, f_{n}\left(x_{1}, \ldots, x_{n}\right) \ldots\right)\right)\right)=f\left(x_{1}, f\left(x_{2}, \ldots, f\left(x_{n-1}, x_{n}\right)\right)\right)$

for all $x, y, z, x_{1}, \ldots, x_{n} \in A$ and for all $f_{n} \in F$.

(2) We choose to call such algebras Birkhoff systems because in his Problem 7 (of [1]) G. Birkhoff has suggested (3) as a possible generalization of the absorption identities in lattices. Again, the name "quasilattices" is motivated by the fact that our "distributive quasilattices" (in which, of course, Q4 and Q4" are now superfluous) are exactly the same distributive quasilattices as described in [5] and consequently our representation theorem (Lemma 3) automatically covers Theorem 3 of [6]. Quasilattices and regular equations were studied by the author in [4] and [5] under the names "compatible systems" and "natural equations". 
The following connection between $P$-functions and the notion of the sum of a direct system of algebras formulated in [7] is of fundamental importance to us:

Plonka's Theorem. Let $\mathfrak{\Re}$ be an equational class of algebras and let $\mathfrak{A} \in \mathfrak{R}$. Let $g(x, y)$ be a binary function in $\mathfrak{A}$ and let $\mathfrak{R}^{*}$ be that equational class of algebras defined by the equations of $\mathfrak{\AA}$ together with $g(x, y)=x$. Then $g(x, y)$ is a P-function for $\mathfrak{A}$ if and only if $\mathfrak{A}$ is representable as the sum of a direct system of algebras from the class $\mathfrak{\AA}^{*}$.

For the notion of the sum of a direct system of algebras we refer to [7]. For other standard concepts such as polynomials in algebras, free algebras, types of algebras, etc., we refer to [2].

\section{LEMMA 1 [4]. Quasilattices are equationally definable.}

Proof. Let $\mathfrak{A}=\langle A,+, \circ\rangle$ be a quasilattice. By the definition of the relation $R_{+}$, we have $(a+b, b) \in R_{+}$for all $a, b \in A$. Hence, by the compatibility requirement, we get $((a+b) c, b c) \in R_{+}$for all $a, b, c \in A$ which yields the identity

Q5. $(a+b) c+b c=(a+b) c$

and similarly its dual

Q5'. $(a b+c)(b+c)=a b+c$

must also hold in $\mathfrak{A}$. Conversely, an algebra $\mathfrak{A}=\langle A,+, \circ\rangle$ satisfying $\mathrm{Q} 1, \mathrm{Q} 2, \mathrm{Q}$, $\mathrm{Q} 5$ and their duals is, indeed, a quasilattice. The proof is routine.

Let us call the set of equations $\left\{\mathrm{Q} 1, \mathrm{Q} 2, \mathrm{Q} 3, \mathrm{Q} 5, \mathrm{Q} 1^{\prime}, \mathrm{Q} 2^{\prime}, \mathrm{Q}^{\prime}, \mathrm{Q}^{\prime}\right\}$ by $Q$. Note that all the members of $Q$ are regular equations true in all lattices. Our claim is that this set $Q$ generates the set of all regular equations true in all lattices. In the following computational lemma we make use of both the equational and implicational form of the compatiblity property that suits the occasion. This will avoid writing equations of huge length.

Lemma 2. In a quasilattice $\mathfrak{A}=\langle A,+, \circ\rangle$ the following identities are true:

$$
\begin{aligned}
a+b a & =(a+b) a, \\
(a+b)+a b & =a+b, \\
a+a b+c b & =a+c b, \\
x_{1}+x_{2}+x_{1} y & =x_{1}+x_{2}+x_{2} y, \\
a+a(b+c) & =a+a b+a c, \\
x+x(y+y z) & =x+x(z+z y), \\
x+x(y+y z) & =(x+x y)+(x+x y) z, \\
\left(x_{1}+x_{2}\right)+\left(x_{1}+x_{2}\right) y & =x_{1}+x_{1} y+x_{2}=x_{1}+x_{2}+x_{2} y, \\
x_{1}+x_{1}\left(x_{2}+x_{2}\left(x_{1}+x_{2}\right)\right) & =x_{1}+x_{1} x_{2} .
\end{aligned}
$$


Proof.

(3)

$$
\begin{aligned}
& a+b a=a b+a \\
& =(a b+a)(b+a) \quad \text { by } \mathrm{Q5}^{\prime} \\
& =((a b+a)(a+a))(b+a) \text { by Q5' } \\
& =(a b+a)(a(b+a)) \\
& =((a+b) a)(b a+a) \\
& =((a+b) a+b a)(b a+a) \text { by Q5 } \\
& =((a+b) a+b a)(a+b a) \\
& =(a+b) a+b a \\
& \text { by } \mathrm{Q5}^{\prime} \\
& =(a+b) a \\
& \text { by Q5. } \\
& (a+b)+a b=a+b+(b+a b) \\
& =(a+b)+(b(a+b)) \quad \text { by (3) } \\
& =((a+b)+b)(a+b) \quad \text { by (3) } \\
& =a+b \text {. } \\
& a+a b+c b=(c b+a)+a b \\
& =(c b+a)(b+a)+a b \text { by Q5'. }
\end{aligned}
$$

Now $(c b+a, a) \in R_{+}$and $(b+a, b) \in R_{+}$and consequently $((c b+a)(b+a), a b) \in R_{+}$ which yields (5).

$$
\begin{aligned}
x_{1}+x_{2}+x_{1} y & =x_{1}+\left(x_{2}+x_{1} y\right) \\
& =x_{1}+\left(x_{2}+x_{2} y+x_{1} y\right) \quad \text { by }(5)
\end{aligned}
$$

and since the latter expression is symmetric in $x_{1}$ and $x_{2}$ we get (6).

$$
\begin{aligned}
a+a b+a c & =a+a b+a b c & & \text { by (6) } \\
& =a+a b(a b+c) & & \text { by (3) } \\
& =a+a b(b+c)(a b+c) & & \text { by Q5' } \\
& =a+a b(b+c)(b+c+c) & & \text { by (6') } \\
& =a+a b(b+c) & & \\
& =a+a(b+c)+a b(b+c) & & \text { by (5). }
\end{aligned}
$$

Now

$$
\begin{aligned}
(b+c)+b(b+c) & =((b+c)+b)(b+c) \text { by }(3) \\
& =b+c
\end{aligned}
$$

and hence $(b+c, b(b+c)) \in R_{+}$. 
So we also have $(a(b+c), a b(b+c)) \in R_{+}$and hence we obtain $a+a b+a c$ $=a+a(b+c)$ which is (7).

$$
\begin{array}{rlr}
x+x(y+y z) & =x+x y(y+z) & \text { by (3) } \\
& =x+x(y+z)+x y(y+z) & \text { by (5) } \\
& =(x+y+z) x+x y(y+z) . &
\end{array}
$$

Now $(x+y+z, y+z) \in R_{+}$and $(x+y+z, y) \in R_{+}$and so $(x+y+z, y(y+z)) \in R_{+}$ which in turn implies that $(x(x+y+z), x y(y+z)) \in R_{+}$and so we have $x+x(y+y z)$ $=(x+y+z) x$. This expression being symmetric in $y$ and $z$ we get (8).

$$
\begin{array}{rlrl}
(x+x y)+(x+x y) z & =x+x y+x(x+y) z & & \text { by (3) } \\
& =x+x(y+(x+y) z) & & \text { by (7) } \\
& =(x+y+(x+y) z) x & & \text { by (3) } \\
& =(x+y+z)(x+y) x & & \text { by (3) } \\
& =x(x+y)(x+(y+z)) & \\
& =x(x+y(y+z)) & & \text { by (7') } \\
& =x+x y(y+z) & & \text { by (3) } \\
& =x+x(y+y z) & & \text { by (3). }
\end{array}
$$

By (6) we have

$$
x_{1}+t+x_{1} y=x_{1}+t+t y .
$$

Putting $t=x_{1}+x_{2}$ in the above, we get $x_{1}+x_{2}+x_{1} y=x_{1}+x_{2}+\left(x_{1}+x_{2}\right) y$ which yields one part of (10). The other part follows by (6) once again.

$$
\begin{array}{rlrl}
x_{1}+x_{1}\left(x_{2}+x_{2}\left(x_{1}+x_{2}\right)\right) & =x_{1}+x_{1}\left(x_{2}+x_{1}+x_{2}\right) x_{2} & & \text { by }(3) \\
& =x_{1}+x_{1}\left(x_{1}+x_{2}\right) x_{2} & \\
& =x_{1}+x_{1} x_{2} & & \text { by }\left(4^{\prime}\right)
\end{array}
$$

and this completes the proof of the lemma.

REMARK. It is clear that (8), (9), (10), (11) and the duals of (10) and (11) along with (3) shows that the binary function $f(x, y)=x+x y$ defines a partition function in any quasilattice.

LEMMA 3. An algebra $\mathfrak{A}=\langle A,+, \circ\rangle$ is a quasilattice if and only if it is the sum of a direct system of lattices.

Proof. If $\mathfrak{A}$ is representable as the sum of a direct system of lattices $\left\{\mathfrak{A}_{i}\right\}$ then all regular equations true in all the $\mathfrak{A}_{i}$ are satisfied in $\mathfrak{A}$ (Theorem 1 of [7]). By our Lemma 1, the defining equations $Q$ of quasilattices are all regular and are valid in all lattices and hence the members of $Q$ are valid in $\mathfrak{A}$ which proves that $\mathfrak{A}$ is a quasilattice. 
Conversely, let $\mathfrak{A}=\langle A,+, 0\rangle$ be a quasilattice. Since the binary function $f(x, y)=x+x y$ is a partition function in $\mathfrak{A}$, by Plonka's Theorem, it is the sum of a direct system of algebras $\left\{\mathfrak{A}_{i}=\left\langle A_{i},+, \circ\right\rangle\right\}$ satisfying the axioms

$$
Q^{*}=Q \cup\{x+x y=x\}
$$

and hence, by our Lemma 2 (equation (3)), each $\mathfrak{A}$ is a lattice $\left(^{3}\right)$. This completes the proof of the lemma.

Corollary 1 (J. PlonKa [6]). Every distributive quasilattice (i.e. a quasilattice in which the distributive laws $a(b+c)=a b+a c$ and $a+b c=(a+b)(a+c)$ hold $\left({ }^{4}\right)$ is the sum of a direct system of distributive lattices.

THEOREM 1. The identities $Q$ form a base for the set of all regular equations of lattices.

Proof. Let $\Re$ denote the set of all regular equations true in all lattices. It is clear that $Q \subset \Re$ and that $Q$ is independent (in the appendix we give a detailed proof of this). Let $\rho$ be an arbitrary member of $\Re$. Let $[\rho]$ be the class of all algebras of type $\langle 2,2\rangle$ satisfying $\rho$. Let $\mathfrak{A}$ be an arbitrary quasilattice. By Lemma $3, \mathfrak{A}$ is the sum of a direct system of lattices, and hence $\rho$, being a regular lattice equation, is satisfied in $\mathfrak{A}$. Thus $\mathfrak{A} \in[\rho]$. This shows that the class of models of $Q$ is a subclass of the class of all models of $\rho$. But it is well known that this is the same as " $Q \Rightarrow \rho$ ". This completes the proof of the theorem.

\section{Applications.}

3.1. The following notation will be useful in this section. Let us agree to call an algebra $\mathfrak{A}=\langle A,+, \circ\rangle$ a bi-semilattice if it satisfies $\mathrm{Q} 1, \mathrm{Q} 2, \mathrm{Q} 3$ and their duals. Let $\mathfrak{B}$ denote the class of all bi-semilattices, $\mathfrak{Q}$-the class of all quasilattices, $\mathfrak{L}$-the class of all lattices, $\subseteq$ - the class of all semilattices (thought of, for purposes of this section as algebras of type $\langle 2,2\rangle$ thus: a semilattice is a bi-semilattice $\mathfrak{A}=\langle A,+, 0\rangle$ which further satisfies the identity $a+b=a b$ ). Let Id $(\mathfrak{X})$ denote the set of all identities true in all members of $\mathfrak{X}$. Now consider the lattice of equational classes of algebras of type $\langle 2,2\rangle$ (see for example [2, p. 172]) - call it $\Re$. It is clear that in this lattice, $\mathfrak{Q} \supset \mathfrak{L}, \mathfrak{Q} \supset \mathfrak{S}$ and that $\mathfrak{L} \wedge \mathfrak{S}=\mathfrak{D}$-the trivial equational class of oneelement algebras $\left({ }^{5}\right)$. Now, let $\mathfrak{X}$ be an arbitrary member of $\mathfrak{A}$ containing both $\mathfrak{R}$

$\left({ }^{3}\right)$ In fact, it is easy to show that a quasilattice satisfying some nonregular identity is a lattice ([4], [5]). Again, in presence of any one of the absorption laws, any one (but not both) of the compatibility properties could be removed to arrive at lattices. Thus our system $Q$ leads to a plethora of new axiom-systems for lattices.

( $\left.{ }^{4}\right)$ It has been shown in [4] and [5] that, as in lattices, validity of one distributive law in a quasilattice is equivalent to the validity of the other.

$\left(^{5}\right)$ Reformulation of the familiar statement that in a lattice $A$ if, for some elements $a$, $b \in A, a+b=a b$ then $a=b$. 
and $\mathfrak{S}$. Then it is well known that $\operatorname{Id}(\mathfrak{X}) \subseteq \operatorname{Id}(\mathfrak{L}) \cap \operatorname{Id}(\mathfrak{S})$, but the identities of $\mathfrak{S}$ being all regular, we have, by Theorem 1 , Id $(\mathfrak{X}) \subseteq \operatorname{Id}(\mathfrak{Q})$, or equivalently $\mathfrak{X} \supseteq \mathfrak{Q}$. Thus we have $\mathfrak{L} \vee \mathfrak{S}=\mathfrak{Q}$. In other words, we have proved the following

THEOREM 2. The class of all quasilattices is the smallest equational class of algebras of type $\langle 2,2\rangle$ containing both the class of all lattices and the class of all semilattices (viewed from the above sense).

Let " $f=g$ " be a regular identity which is not strictly consistent (see [2, p. 173]) with the lattice identities. Then it is clear that $Q \cup\{f=g\} \Rightarrow$ “ $a+b=a b$ ". In view of this, together with the remark of the footnote 2 and the results of $\S 2$, it can be shown that the lattice of all equational classes of quasilattices is isomorphic to the direct product of the lattice of all equational classes of lattices and the two-element chain and hence, by a result of B. Jónsson [3], it is distributive.

3.2. In $\$ 2$ we noticed that in bi-semilattices the compatibility property (Q4 and Q4') was characterized by a set of ternary lattice identities, but in lattices themselves this property is a consequence of binary identities (e.g. the absorption laws). Thus the question arises whether Q4 and Q4' can be made equivalent to any set of binary identities. The answer is in the negative because it is easy to give examples of bisemilattices $\mathfrak{A}=\langle A,+, \circ\rangle$ such that every subalgebra of $\mathfrak{A}$ generated by two elements is a quasilattice but $\mathfrak{A}$ itself is not a quasilattice. However, it will be illuminating to give an indirect proof of this negative assertion using a property of free Birkhoff systems which, incidentally, is pressed into service to yield a finite basis for the set of all binary regular lattice equations.

LEMMA 4. FBS(2)-the free Birkhoff system on two generators is a quasilattice.

Proof. Let $a$ and $b$ be the two generators. Using Q1, Q2, Q3, their duals and (3) it is easy to show (actual computation is made in the proof of Theorem 3 below) that FBS(2) contains just six elements, namely $\{a, b, c=a+b, d=a b, e=a+a b$, $f=b+a b\}$ and the operations are defined by the "meet" of the following semilattice diagrams:
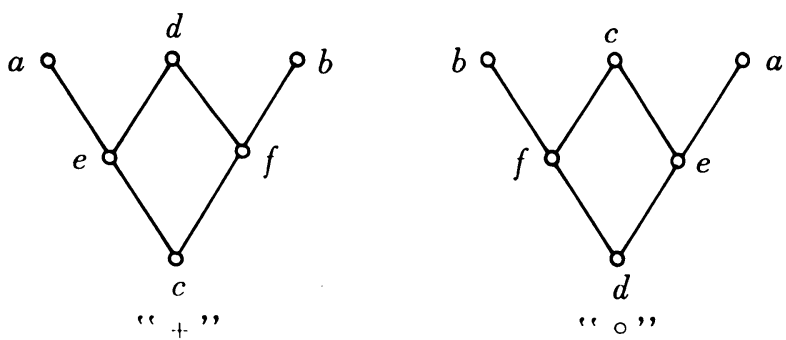

Now to show that this algebra is a quasilattice one need only verify the validity of say, the identity Q5 in FBS(2) which is quite routine. But we will avoid this verification by showing that FBS(2) is the sum of a direct system of lattices and then 
appeal to our Lemma 3 to show that this is indeed a quasilattice. Let $I=\{1,2,3\}$ be an index set with $1 \leqq 3,2 \leqq 3,1 \$ 2,2 \$ 1$. Let $A_{1}$ and $A_{2}$ be two copies of the one-element lattice, say $\{a\}$ and $\{b\}$ respectively and let $A_{3}$ be the lattice

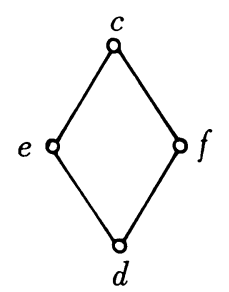

and let $\varphi_{13}: A_{1} \rightarrow A_{3}$ be defined as $a \varphi_{13}=e$ and let $\varphi_{23}: A_{2} \rightarrow A_{3}$ be defined as $b \varphi_{23}=f$. It is clear that $\left\{A_{i}\right\}_{i \in I}$ is a direct system of lattices. If $\mathfrak{A}=\langle A,+, 0\rangle$ is the quasilattice which represents the sum of the system $\left\{A_{i}\right\}$ then $A=\{a, b, c, d, e, f\}$ where, for example, $a+b=a \varphi_{13}+b \varphi_{23}=e+f=c, a b=e f=d$, etc. In fact, $\mathfrak{A}$ is isomorphic to $\operatorname{FBS}(2)$ and this completes the proof of the lemma.

COROLlaRY 2. In bi-semilattices, the compatibility property $\mathrm{Q} 4$ and $\mathrm{Q} 4$ ' cannot be made equivalent to any set $\Sigma$ of binary lattice identities.

Proof. If possible, let $\Sigma$ be a set of binary lattice identities such that a bisemilattice $\mathfrak{A}$ will be a quasilattice if and only if all the members of $\Sigma$ hold in $\mathfrak{A}$. Let $\mathfrak{A}=\langle A,+, \circ\rangle$ be an arbitrary Birkhoff system and let $S$ be an arbitrary subalgebra of $\mathfrak{A}$ generated by two elements. Now $S$ itself is a Birkhoff system and it is generated by two elements and hence it is a homomorphic image of FBS(2). But by the previous lemma, $\mathrm{FBS}(2)$ is a quasilattice and since being a quasilattice is equational (Lemma 1), $S$ is a quasilattice. Thus, by our assumption, every member of $\Sigma$ holds in $S$. $S$ being an arbitrary subalgebra of $\mathfrak{A}$ generated by two elements and $\Sigma$ being a collection of binary identities, we see that every member of $\Sigma$ holds in $\mathfrak{A}$ which shows that $\mathfrak{A}$ is a quasilattice. In other words, we have proved that every Birkhoff system is a quasilattice. This is the desired contradiction since there are examples of Birkhoff systems which are not quasilattices. One such example would be $\mathfrak{A}=\langle\{a, b, c\} ;+, 0\rangle$ where the operations are defined by the following tables.

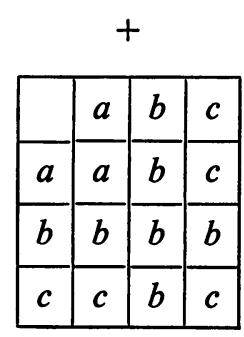

\begin{tabular}{|l|l|l|l|}
\hline & $a$ & $b$ & $c$ \\
\hline$a$ & $a$ & $a$ & $a$ \\
\hline$b$ & $a$ & $b$ & $b$ \\
\hline$c$ & $a$ & $b$ & $c$ \\
\hline
\end{tabular}


Here $a \cdot b=a$ and hence $(a, b) \in R_{\circ}$ but $(a+c, b+c)=(c, b) \notin R_{\circ}$ since $c \cdot b \neq c$. It is easy to verify that $\mathfrak{A}$ is a Birkhoff system (also it satisfies the other compatibility condition). This completes the proof of the corollary.

Now, if one takes a close look at the computation of the elements of FBS(2) then it will become clear that one does not actually require the full power of the associativity but only needs the following mild forms (it is easy to give examples of algebras satisfying these but not the associative laws):

$$
\begin{aligned}
& a+(a+b)=a+b, \\
& (a+b)+a b=a+b, \\
& a(a+b)+b(a+b)=a+b, \\
& \text { (14') }(a+a b)(b+a b)=a b \text {, } \\
& a+(b+a b)=a+b, \\
& a(b(a+b))=a b .
\end{aligned}
$$

Thus one could conclude that the free $\Sigma$-algebra generated by two elements-where $\Sigma$ is the set of (thirteen) binary regular lattice identities $\{\mathrm{Q} 1, \mathrm{Q} 2,(3),(12),(13),(14)$, (15) and their duals\}, is isomorphic to $\operatorname{FBS}(2)$ and hence is a quasilattice. Let $\rho$ be any binary regular lattice identity. Let $\mathfrak{A}$ be any $\Sigma$-algebra and let $S$ be a subalgebra of $\mathfrak{A}$ generated by two elements. By the above observation it is a quasilattice and hence, by a special case of Theorem $1, \rho$ is valid in $\mathfrak{A}$ which shows that every model of $\Sigma$ is a model of $\rho$. In other words, we have completed the proof of the following

THEOREM 3. The set of all binary regular identities true in all lattices is finitely based.

In conclusion, I wish to express my appreciation and thanks to Professor G. Grätzer for having useful conversation with him which helped me to shape the formulations while writing this paper.

Appendix. Here we will prove the independence of the identities $Q:\left\{\mathrm{Q} 1, \mathrm{Q} 2, \mathrm{Q} 3, \mathrm{Q} 5, \mathrm{Q}^{\prime}, \mathrm{Q} 2^{\prime}, \mathrm{Q}^{\prime}, \mathrm{Q}^{\prime}\right\}$. In view of the duality it is sufficient if we prove that unprimed identities are independent:

Q1. $a+a=a$ is independent from the rest.

Let $\mathfrak{U}=\langle A,+, \circ, 0\rangle$ be such that $\circ$ is a semilattice operation in $A, a \cdot 0=0$ $\forall a \in A$ and let $a+b=0 \forall a, b \in A$.

Q2. $a+b=b+a$ is independent from the rest.

Let $\mathfrak{A}=\langle A,+, \circ\rangle$ be such that $|A|>1$ and let $a+b=a \forall a, b \in A$ and $\circ$ be any semilattice operation.

Q3. Let $R$ denote the set of real numbers and let $A$ be the set of all subsets of $R$. Consider the algebra $\mathfrak{A}=\langle A,+, \circ\rangle$ where

$$
\begin{aligned}
b+a=a+b=[a, b], & \text { the closed interval containing } a \text { and } b \\
& \text { if } a \text { and } b \text { are one-point subsets of } R, \\
& =a \cup b \quad \text { otherwise, }
\end{aligned}
$$

and

$$
a \circ b=a \cap b \text { for all } a, b \in A \text {. }
$$


It is clear that if $p<q<r$ then $p+(q+r) \neq(p+q)+r$. It is easy to check the validity of other identities of $Q$. In fact, in this algebra every binary lattice identity is true and the verification of Q5 and Q5' is immediately obtained once we notice that $\mathrm{Q} 5\left(\mathrm{Q}^{\prime}\right)$ is a consequence of $\mathrm{Q} 4\left(\mathrm{Q}^{\prime}\right)$ and binary lattice identities. (This example, as well as the previous ones, can be so chosen that $|A|$ is finite.)

Q5. See the example given under Corollary 2.

Added in proof (December 31, 1970). (1) The remark following Theorem 2, namely the description of the lattice of all equational subclasses of quasilattices has been generalized by H. Lakser, C. R. Platt and the author to arbitrary equational classes of algebras with mild restrictions (see Abstract \#70T-A205, Notices Amer. Math. Soc. 17 (1970), p. 945). (2) The claim made with respect to Example 3 in the Appendix above is incorrect. It does not satisfy Q5'. Hence the question whether $Q$ is independent is not settled here. (3) Due to a recent result of the author, any finitely based equational theory of idempotent algebras of type $\langle m, n\rangle$ with $m, n \geqq 2$ is two-based. Hence $Q$ can be made equivalent to a set of two-identities and it is clear that $Q$ is not one-based.

\section{REFERENCES}

1. G. Birkhoff, Lattice theory, 2nd rev. ed., Amer. Math. Soc. Colloq. Publ., vol. 25, Amer. Math. Soc., Providence, R. I., 1948. MR 10, 673.

2. G. Grätzer, Universal algebra, The University Series in Higher Math., Van Nostrand, Princeton, N. J., 1968.

3. B. Jónsson, Algebras whose congruence lattices are distributive, Math. Scand. 21 (1967), 110-121. MR 38 \#5689.

4. R. Padmanabhan, Studies in the axiomatics of groups and lattices, Doctoral Dissertation, Madurai University, 1967.

5. —_ Link-laws in lattice theory, Madurai Univ. J. 1 (1970).

6. J. Plonka, On distributive quasi-lattices, Fund. Math. 60 (1967), 191-200. MR 36 \#85.

7. - On a method of construction of abstract algebras, Fund. Math. 61 (1967), 183-189. MR 37 \#1294.

8. - Some remarks on sums of direct systems of algebras, Fund. Math. 62 (1968), 301308. MR 38 \#2073.

UNIVERSITY OF MANITOBA, WINNIPEG, CANADA 